\title{
A Two-Photon Single-Laser Optical Data Storage System in a Multilayer Structure
}

\author{
Qun Hao, Xuemin Cheng ${ }^{1, *}$, Xinnan Hou ${ }^{1}$ and Jianshe $\mathrm{Ma}^{1}$ \\ School of Optoelectronics, Beijing Institute of Technology, No. 5 Zhongguancun South Street, \\ Haidian District, Beijing, 100081, China \\ ${ }^{1}$ Graduate School at Shenzhen, Tsinghua University, \\ Univ. Town, Xili, Nanshan District, Shenzhen, 518055, China
}

(Received July 2, 2014; accepted March 23, 2015)

Key words: optical data storage, single-laser system, two-photon system, multilayer structure

In this work, a two-photon recording system in a 50-layer structure is designed with each recording layer containing $4.7 \mathrm{~GB}$ of data, similar to the capacity of a singlelayer digital versatile disc, with the total capacity becoming $235 \mathrm{~GB} /$ disc. By the use of a single-laser system to realize recording and reading, a multilayer disc structure is applied in which the groove structure is set on the substrate for servo control; the laser is separated by a polarized beam splitter (PBS) into two parts, one for recording and the other for servo control in the optical system. The simulation results show the feasibility of high-density optical data storage using a single laser in a multilayer structure. The experimental platform is set up in which the focus error signal is verified.

\section{Introduction}

To develop new technologies to enlarge the capacity of an optical data storage system, two-photon multilayer ${ }^{(1-3)}$ technologies that allow for volumetric storage have been proposed. Exploiting the two-photon fluorescence effect for volume storage, $\mathrm{Gu}$ proposed the high-density multilayer recording in one disc with an equivalent capacity of $298 \mathrm{~GB}^{(3)}$ In 2008 , Walker et al. presented the materials and methods for 1 Tbyte of data storage in 200 layers inside the volume of one disc. ${ }^{(4)}$ In 2009, true five-dimensional optical recording with an equivalent capacity of $10^{12} \mathrm{bits} / \mathrm{cm}^{3}$ was investigated using the LSPR effect of gold nanorods, ${ }^{(5)}$ including the information of wavelength and polarization as well as three spatial dimensions. Two-photon methods have been proven to be efficient for high-density volume storage. In most of the present two-photon optical data storage systems, there are two lasers to realize recording and reading separately. ${ }^{(6)}$ The opto-mechanical platform, however, is complex and the disc

${ }^{*}$ Corresponding author: e-mail: cheng-xm@mail.tsinghua.edu.cn 
will vibrate when rotating and the servo system will fail to realize stable recording and reading when the number of layers is increased. In Walker's prototype of 1 Tbyte storage system, one laser was applied for recording and reading, while scanning in the multilayer structures with each layer separated by $5 \mu \mathrm{m}$ is realized by controlling the disk with a motorized stage.

In this work, a single-laser two-photon optical data storage system is proposed. The disc structure in the system is specified as the groove structure on the substrate, and the recording layers and space layers are alternately coated on the substrate. The focus positions of the recording beam and servo beam are independently controlled by changing the positions of the objective lens and the top mirror. The top mirror is controlled with the motorized stage, while the objective lens is controlled independently at different focus positions for focusing and layer selection. Thus, the corresponding reading and recording characteristics of the storage system are designed to possess good linearity and a large linear region, which can be properly integrated into the standard digital versatile disc (DVD) servo platform.

\section{Materials and Methods}

\subsection{Layout}

The single-laser two-photon optical data storage system contains a multilayer structure, a laser beam separation module, a disc-focusing servo module, and a fluorescence signal reading module, as shown in Fig. 1.

\subsection{Multilayer disc structure}

In this work, a multilayer disc structure is applied with the groove structure on the substrate as the servo layer, then alternately coated with a recording layer and a spacer layer on it, and then finally protected by the cover layer on top, as shown in Fig. 2(a). According to the present level of technology, the depth of the substrate and cover layer

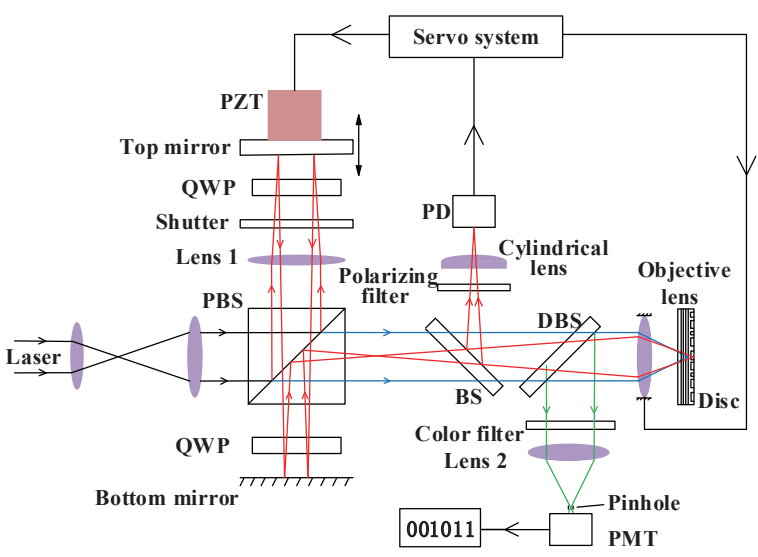

Fig. 1. (Color online) Design of a single-laser two-photon optical data storage system. 


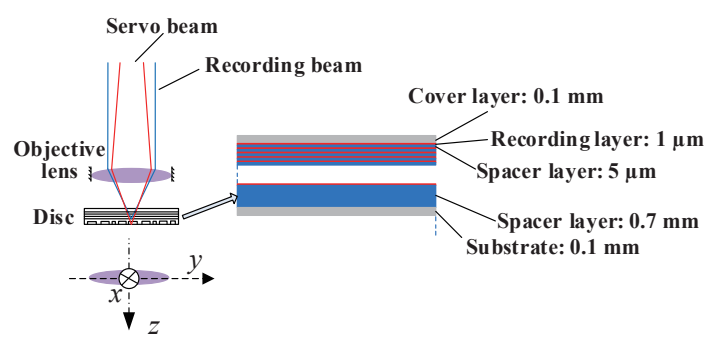

(a)

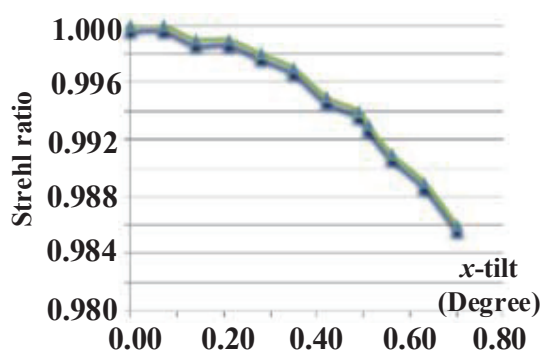

(b)

Fig. 2. (Color online) (a) Schematic drawing of multilayer disc structure and (b) Strehl ratio for objective lens.

is set to $0.1 \mathrm{~mm}$, and the recording layer to $1 \mu \mathrm{m}$. The depth of the spacer layer between the adjacent recording layers is set to $5 \mu \mathrm{m}$, mainly considering the crosstalk. ${ }^{(7)}$ The diameter of the disc is $120 \mathrm{~mm}$, and the total thickness is $1.2 \mathrm{~mm}$, obeying the DVD specification. The number of recording layers is 50 , and each layer contains $4.7 \mathrm{~GB}$ of data similar to the capacity of a single-layer DVD system. The total capacity is 235 GB in this type of multilayer disc.

In the system, it is designed that the wavefront error of the objective with NA of 0.6 is below $0.03 \lambda_{\text {rms }}$ when the light spot is focused on the corresponding layers without an $x$-tilt [the coordinate system defined in Fig. 2(a)]. The Strehl ratio for focusing will decrease with increasing $x$-tilt angle, as shown in Fig. 2(b). It shows that the value of the Strehl ratio is smaller than 0.998 when the $x$-tilt is $<0.15^{\circ}$.

\subsection{Separation of the recording beam and servo beam}

To record in the new type of multilayer disc, the laser beam is separated, one for recording and the other for focusing and tracking, as shown in Fig. 3(a). A polarized beam splitter (PBS) is used to separate a laser beam into two perpendicular polarized beams. ${ }^{(8)}$ The recording beam and servo beam are focused by one objective lens. The recording beam (p-polarized beam) passes through the PBS and is focused on the recording layer by the objective lens. The focus position of the recording beam can be controlled by changing the position of the objective lens.

The servo beam (s-polarized beam) is reflected from the PBS to lens 1 (a spherical lens), the shutter, and the quarter-wave plate (QWP). Lens 1 is placed between the two mirrors to change the incident angle of the servo beam at the objective lens. After being reflected by the top mirror, the beam passes through the PBS to the QWP, shutter, and bottom mirror. As the QWP changes the polarization status, the servo beam reflected by the bottom mirror is finally reflected from the PBS again and is focused on the servo layer on the substrate after passing through the objective lens.

The focus position of the servo beam is controlled by changing the position of the top mirror. By using the polarization properties of the recording beam and the servo beam, the two beams can be controlled independently at different focus positions. The focus positions of the two beams are independently controlled by changing the positions of the objective lens and the top mirror. 


\subsection{Disc-focusing servo}

The focusing servo module includes a transflective mirror, a polarizer, a cylindrical lens, and a four-quadrant photodetector (PD), as shown in Fig. 3(b). It is necessary to realize stable recording and reading by maintaining a constant distance between the objective lens and the disc.

After being reflected by the disc, the servo beam is focused on PD by the objective lens. The transreflective mirror is used to change the optical path of the reflected servo beam. The recording beam is filtered by the polarizer so that only the servo beam is detected at PD. The servo beam is modulated during the recording process, as the cylindrical lens is used to apply the astigmatism method to the disc-focusing servo. ${ }^{(9)}$ When the servo beam is in focus, the spot at PD is circular. Otherwise, the spot is oval. The focus error (FE) signal can be calculated from the PD output. Correspondingly, the sign and value of FE will change depending on whether the servo beam is in focus or not, as shown in Fig. 4. Good linearity and a large linear region are the preconditions for achieving high-precision focusing servo.

\subsection{Fluorescence signal reading}

The fluorescence signal reading module includes a dichroic mirror, a color filter, a spherical lens (lens 2), a pinhole, and a photomultiplier tube (PMT), as shown in Fig. $5(\mathrm{a})$.

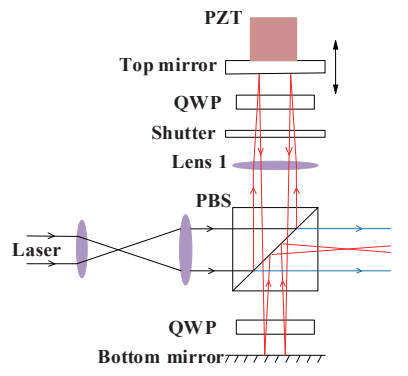

(a)

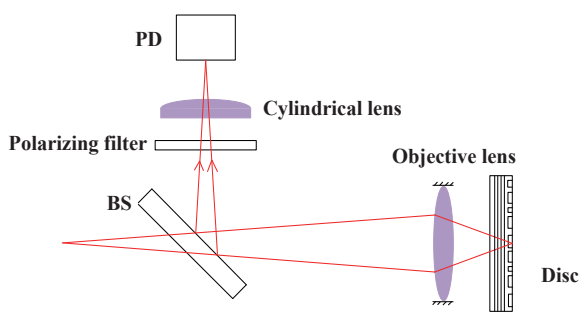

(b)

Fig. 3. (Color online) (a) Separation of the recording beam and servo beam and (b) disc-focusing servo module.

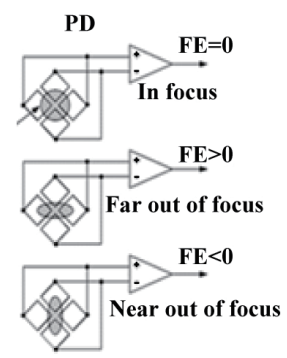

(a)

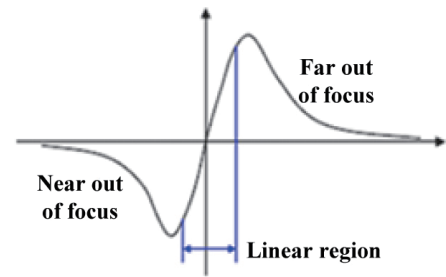

(b)

Fig. 4. (Color online) (a) Spot form at the PD and (b) FE signal curve. 


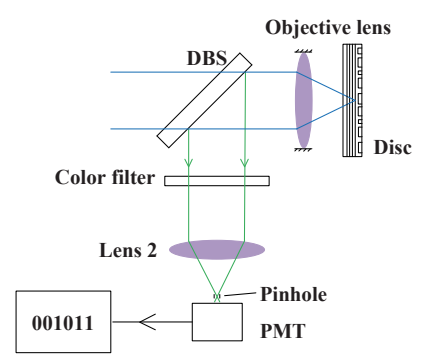

(a)

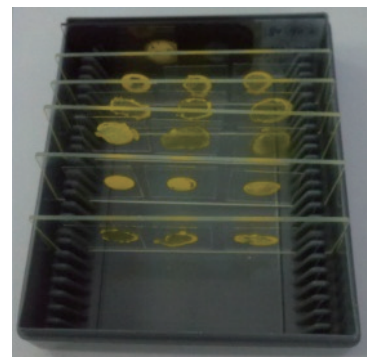

(b)

Fig. 5. (Color online) (a) Fluorescence signal reading module and (b) two-photon material deposition on the plate.

Fluorescence (short wavelength) will be excited out from the two-photon material, shown in Fig. 5(b), when the recording beam (long wavelength) focuses on the recording layer of the disc through the objective lens. The fluorescence beam was collected by the same objective lens and directed to the PMT by the dichroic mirror, converted into an electrical signal, and normalized to zero and unit variance. The remaining recording beam is filtered out by the color filter and the pinhole is used to filter out noise beams from neighboring layers by confocal microscopy. ${ }^{(10)}$

\subsection{Method of changing recording and reading positions}

In the optical data storage system, the recording and reading positions are changed between different recording layers by moving the top mirror and the objective lens. ${ }^{(1)}$ Figure 6 shows the position parameters of optical elements; the focal lengths of lens 1 and lens 2 are $f_{1}$ and $f_{2}$, respectively.

The servo beam passes lens 1 twice and is focused on the servo layer by the objective lens. According to the imaging formula and the geometric relationship, eq. (1) is established and the relationship between $x_{n}$ and $n$ in eq. (2) is achieved, as it is a oneto-one correspondence between the position of the top mirror and the layer number $n$, satisfying the conjugate imaging principle.

$$
\begin{gathered}
\left\{\begin{array}{l}
l_{1}^{\prime}=f_{1} ; l_{2}=f_{1}-2 x ; 1 / l_{2}^{\prime}-1 / l_{2}=1 / f_{1} ; \\
l_{2}^{\prime}=a+2 b+d+l_{3} ; 1 / l_{3}^{\prime}-1 / l_{3}=1 / f_{2} ;
\end{array}\right. \\
x_{n}=\frac{\left\{2 a+4 b+2\left[d_{1}+0.006(n-1)\right]-f_{1}^{\prime}+\left\{2 f_{2}^{\prime}\left[l_{31}^{\prime}-0.006(n-1)\right]\right\} /\left\{f_{2}^{\prime}-\left[l_{31}^{\prime}-0.006(n-1)\right]\right\}\right\} f_{1}^{\prime}}{2\left\{a+2 b+\left[d_{1}+0.006(n-1)\right]-f_{1}^{\prime}+\left\{f_{2}^{\prime}\left[l_{31}^{\prime}-0.006(n-1)\right]\right\} /\left\{f_{2}^{\prime}-\left[l_{31}^{\prime}-0.006(n-1)\right]\right\}\right\}}
\end{gathered}
$$

The initial values are set as $a=4 \mathrm{~mm}, b=5.5 \mathrm{~mm}, d_{1}=16 \mathrm{~mm}, l_{31}^{\prime}=4 \mathrm{~mm}, f_{1}^{\prime}=50$ $\mathrm{mm}, f_{2}^{\prime}=3 \mathrm{~mm}$, so that $x_{1}=9.677 \mathrm{~mm}, x_{2}=9.740 \mathrm{~mm}, \ldots, x_{50}=13.828 \mathrm{~mm}$. Thus, the minimum movement of the top mirror is $\Delta x_{\min }=0.063 \mathrm{~mm}$, and the maximum movement is $\Delta x_{\max }=x_{50}-x_{1}=4.151 \mathrm{~mm}$. The high-precision translation stage is selected using 


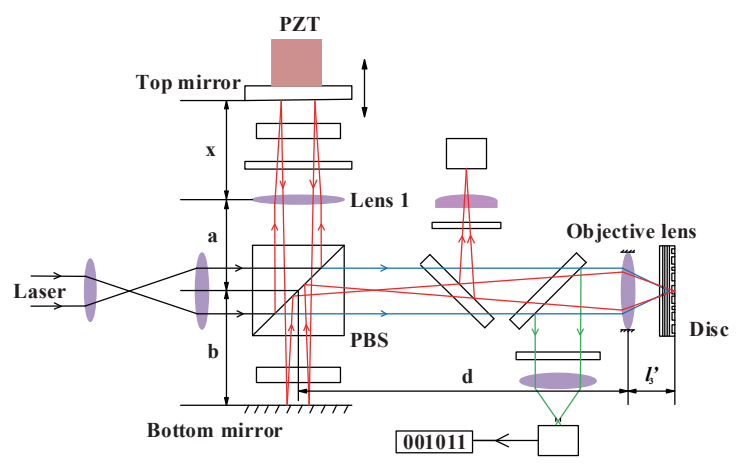

Fig. 6. (Color online) Position parameters of optical elements.

Feinmess KDT180-100-LM. The parameters are shown in Table 1. The translation accuracy of the stage is $1 \mu \mathrm{m}$, satisfying the requirement of coarse servo-tuning. The average speed of $100 \mathrm{~mm} / \mathrm{s}$ is used to calculate the time of layer-changing, $\Delta t$. It shows that the system possesses the characteristic of fast layer-changing with a maximum time of $41.5 \mathrm{~ms}$.

$$
0.6 \mathrm{~ms}=\Delta x_{\min } / 100 \leq \Delta t \leq x_{\max } / 100=41.5 \mathrm{~ms}
$$

\section{Analysis}

\subsection{Focusing error}

The servo beam goes through the cylindrical lens to the PD, when modulated by the groove structure on the servo layer during the recording process, reflected by the disc and collected by the objective lens. The FE signal is calculated using the equation $F E=$ $(A+C)-(B+D) ; A, B, C$, and $D$ represent the four-quadrant output 4-way signals. When the servo beam is in focus, the spot form at the PD is circular, and the distributions of intensity are uniform at the four quadrants, as shown in Fig. 7. In this case, the FE signal is almost zero.

\subsection{Far out of focus}

When the servo beam is far out of focus, the spot form at the PD is oval, and the distributions of intensity are nonuniform at the four quadrants, as shown in Fig. 8(a). In this case, the FE signal is greater than zero. When the servo beam is $+10 \lambda$ out of focus, the spot at the PD is almost a line and FE reaches its maximum value. After that, the spot begins to diverge, and the FE signal's value becomes increasing smaller until almost zero.

\subsection{Near out of focus}

When the servo beam is near out of focus, the spot form at the PD is oval, and the distributions of intensity are nonuniform at the four quadrants, as shown in Fig. 8(b). In 
Table 1

Technical parameters of the stage.

\begin{tabular}{|c|c|c|c|c|c|c|}
\hline $\begin{array}{l}\text { Travel } \\
(\mathrm{mm})\end{array}$ & $\begin{array}{l}\text { Accuracy } \\
\quad(\mu \mathrm{m})\end{array}$ & $\begin{array}{l}\text { Repeatability } \\
(\mu \mathrm{m})\end{array}$ & $\begin{array}{l}\text { Relative deviation } \\
\text { from straightness/ } \\
\text { flatness }(\mu \mathrm{m})\end{array}$ & $\begin{array}{l}\text { Max speed } \\
(\mathrm{mm} / \mathrm{s})\end{array}$ & $\begin{array}{l}\text { Max. acceleration } \\
\left(\mathrm{m} / \mathrm{s}^{2}\right)\end{array}$ & $\begin{array}{c}\text { Permissible loads } \\
F_{x} / F_{y} / F_{\mathrm{z}}(\mathrm{N})\end{array}$ \\
\hline 100 & 1 & $<0.3$ & 1 & 200 & 10 & $15 / 30 / 50$ \\
\hline
\end{tabular}

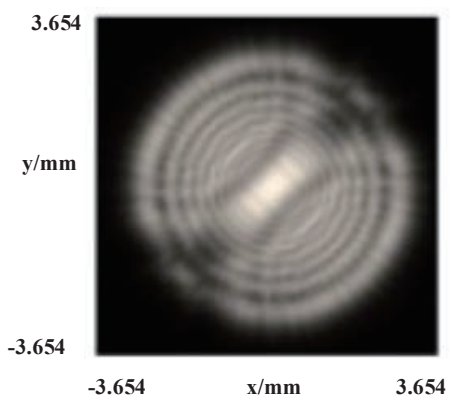

(a)

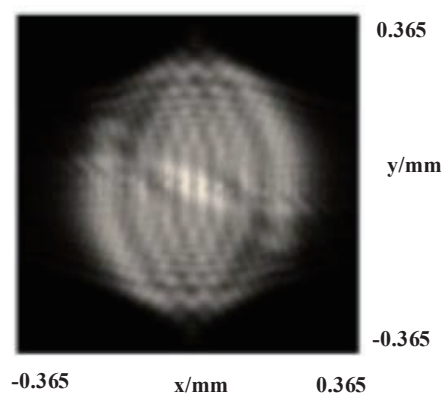

(b)

Fig. 7. Distributions of intensity (a) at the entrance pupil of the cylindrical lens and (b) at the PD.
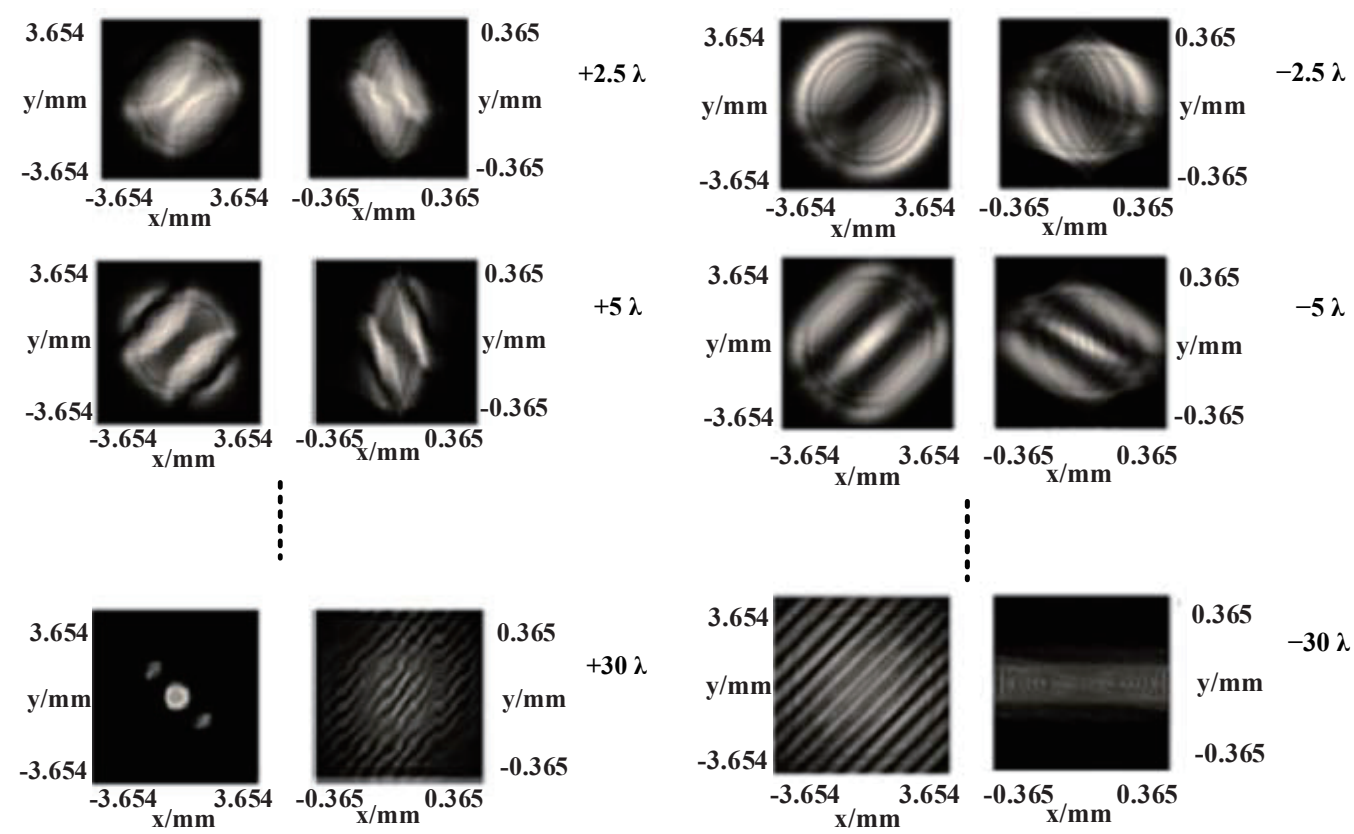

(a)

(b)

Fig. 8. Distributions of intensity for (a) far out of focus and (b) near out of focus with the left column at the entrance pupil of the cylindrical lens and the right column for PD. 
this case, the FE signal is less than zero. When the servo beam is $-25 \lambda$ out of focus, the spot at the PD is almost a line and FE reaches its maximum absolute value. After that, the spot begins to diverge, and the FE signal's absolute value becomes increasing smaller until almost zero.

\subsection{FE signal}

The relationship curve between the FE signal and the defocus distance is shown in Fig. 9(a). As shown in Fig. 9(b), $R^{2}=0.9882$ is achieved at the large region $(-7.5 \lambda, 10 \lambda)$. It indicates that the FE signal has a good linear region for high-precision focusing servo.

\subsection{Servo control modules}

The servo control module is shown in Fig. 10. The parameters for the preamplifier and calculator circuits are designed in relation to the movement of the top mirror, which is controlled using a piezoelectric ceramic sensor (PZT). In the process of rotation disc vibration, the FE signals are used as the feedback to control the movements of the PZT as well as the actuator.

\subsection{Servo precision for focus}

The S-curves for the focus servo error, shown in Fig. 11(a), are tested when the input of the focus coil is a triangular wave. Its slope of the linear region is

$$
K_{\mathrm{S}}=\frac{2.00 \times 500 \mathrm{mV}}{0.35 \times 4.00 \mathrm{~ms}}=714.286 \mathrm{mV} / \mathrm{ms},
$$

where the cycle of the triangular wave is $T_{\text {tri }}=685.354 \mathrm{~ms}$, and the peak-to-peak voltage is $V_{\mathrm{pp}}=0.828 \mathrm{~V}$. The sensitivity of the focus coil is $K_{\mathrm{a}}=530 \mu \mathrm{m} / \mathrm{V}$; thus, the sensitivity of the focus servo error is calculated as

$$
K_{\mathrm{FE}}=\frac{K_{\mathrm{S}} T_{\mathrm{tri}}}{2 V_{\mathrm{pp}} K_{\mathrm{a}}}=557 \mathrm{mV} / \mu \mathrm{m} .
$$

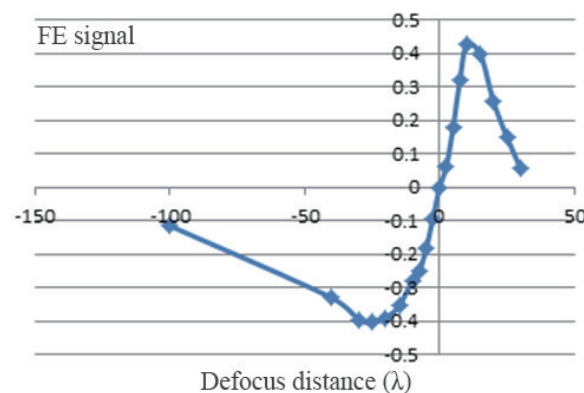

(a)

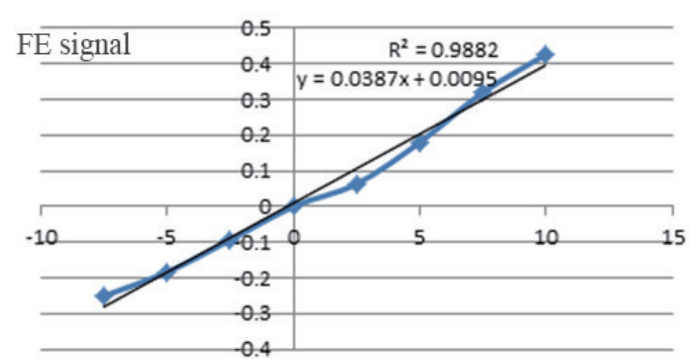

Defocus distance $(\lambda)$

(b)

Fig. 9. (Color online) (a) Relationship curve between FE signal and defocus distance and (b) linear region of the relationship curve between FE signal and defocus distance. 


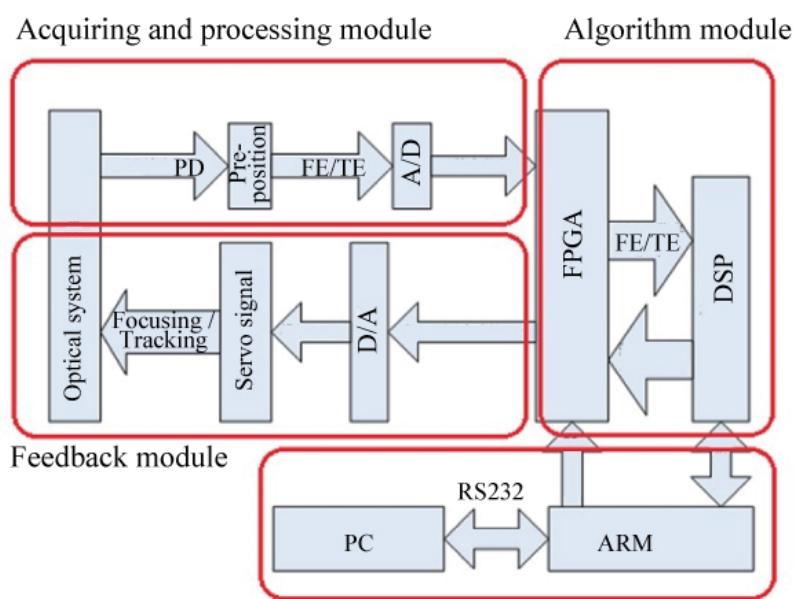

Master controlling module

Fig. 10. (Color online) Servo control modules for the system.

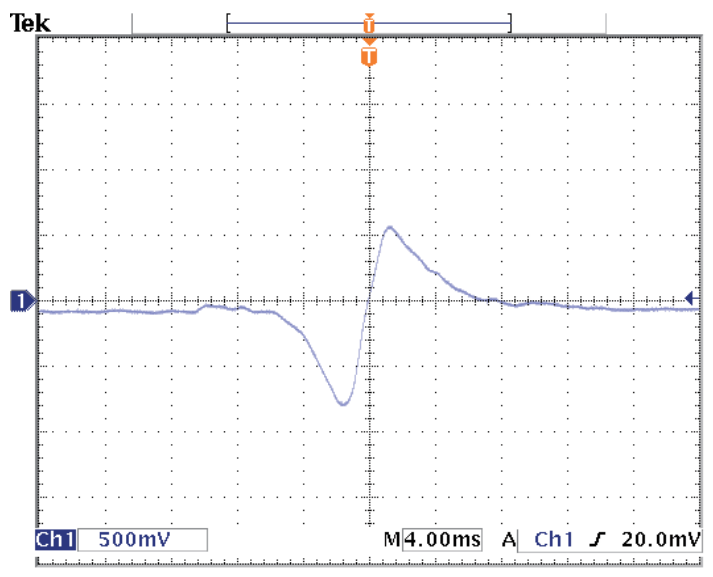

(a)

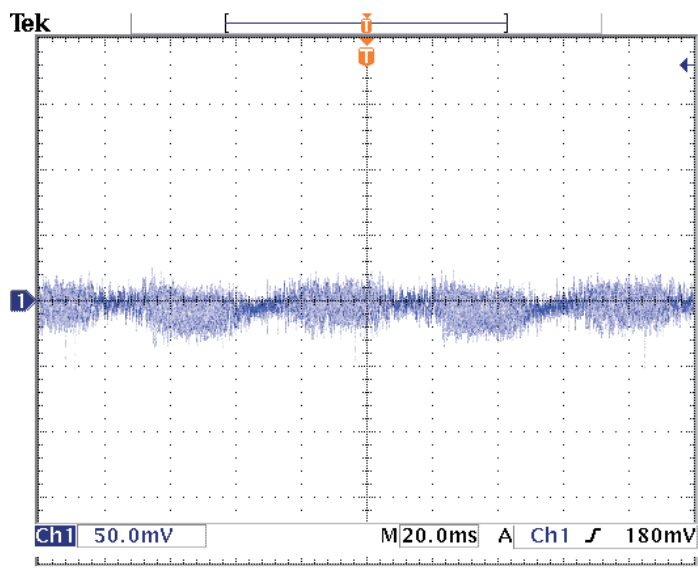

(b)

Fig. 11. (Color online) (a) S-curves for focus servo error and (b) stable focus error signal for closed loop servo.

For the closed loop servo, the stable focus error signal is illustrated in Fig. 11(b). The signal amplitude is $\left|e_{\mathrm{FE}}\right|<50 \mathrm{mV}$ and the focus servo error is calculated using eq. (6). It shows that a stable focus control of an optical disk drive is achieved for a single-layer disk. The servo control of the multilayer disc will be discussed in a future work.

$$
\left|E_{\mathrm{FE}}\right|=\frac{\left|e_{\mathrm{FE}}\right|}{K_{\mathrm{FE}}}<0.090 \mu \mathrm{m}
$$




\section{Conclusions}

In this work, a single-laser two-photon optical data storage system is designed, which has 50 recording layers and a total capacity of $235 \mathrm{~GB} /$ disc. The system has a good linear region, that is, $R^{2}=0.9882$ at the large region $(-7.5 \lambda, 10 \lambda)$ for high-precision focusing servo. The movement accuracy of the top mirror is $1 \mu \mathrm{m}$, satisfying the requirement of coarse servo-tuning. The sensitivity of the focus coil for the objective lens is $K_{\mathrm{a}}=530 \mu \mathrm{m} / \mathrm{V}$, and the sensitivity of the focus servo error is $557 \mathrm{mV} / \mu \mathrm{m}$ for the movement of the objective lens, which satisfies the requirement of the standard DVD servo platform. The capacity of the disc is enlarged by increasing the areal density of each recording layer. Above all, the research shows the feasibility of high-density twophoton optical data storage using a single laser in a multilayer disc. The parameters for the groove structure will be discussed in a future work, by checking the physical size of the recording points and the corresponding fluorescence signals when controlling the laser power.

\section{Acknowledgements}

This research was supported by a grant from the National Natural Science Foundation of China (Nos. 61275003 and 51327005), the Research Fund for Shenzhen Key Laboratory of LED Packaging (ZDSY20120619141243215), and Guangdong Project (No. 2012B091100014). Many thanks to Dr. Xiangping Li (Centre for Micro-Photonics, Faculty of Engineering and Industrial Sciences, Swinburne University of Technology, Hawthorn, Victoria 3122, Australia) for providing the two-photon materials and kind discussion on the preparation of the sample plates.

\section{References}

1 D. A. Parthenopoulos and P. M. Rentzepis: Science 245 (1989) 843.

2 D. Day, M. Gu and A. Smallridge: Opt. Lett. 24 (1999) 948.

3 M. Gu: Photorefractive Fiber and Crystal Devices: Materials, Optical Properties, and Applications VIII, eds. F. T. S. Yu and R. Guo (SPIE, Seattle, 2002) p. 151.

4 E. Walker, A. Dvornikov, K. Coblentz and P. Rentzepis: Appl. Opt. 47 (2008) 4133.

5 P. Zijlstra, J. W. M. Chon and M. Gu: Nature 459 (2009) 410.

6 B. Jiang: Ph.D. Dissertation, Department of Precision Machinery \& Precision Instrumentation (University of Science and Technology of China, Hefei, 2007) p. 37.

7 I. Ichimura, K. Saito, T. Yamasaki and K. Osato: Appl. Opt. 45 (2006) 1794.

8 S. Lee, N. Park, H. Yang, K. Park and Y. Park: J. Opt. 13 (2011) 055403.

9 E. Walker, X. Zheng, F. McCormick, H. Zhang, N. Kim, J. M. Costa and A. Dvornikov: Optical Data Storage 2000, eds. D. Stinson and R. Katayama (SPIE, Whistler, 2000) p. 179.

10 A. Toriumi, S. Kawata and M. Gu: Opt. Lett. 23 (1998) 1924.

11 B. Zhang, J. Ma, L. Pan, X. Cheng and Y. Tang: IEEE Trans. Consum. Electron. 54 (2008) 1743. 